\title{
Recurrence after gross-total resection of low-grade pediatric brain tumors: the frequency and timing of postoperative imaging
}

\author{
Clinical article
}

\author{
Albert H. Kim, M.D., Ph.D., ${ }^{1}$ Elizabeth A. Thompson, A.B., ${ }^{1}$ Lance S. Governale, M.D., ${ }^{2}$ \\ Catalina Santa, ${ }^{3}$ Kevin Cahill, M.D., Ph.D., M.P.H., ${ }^{3}$ Mark W. Kieran, M.D., Ph.D., ${ }^{4}$ \\ Susan N. Chi, M.D. ${ }^{4}$ Nicole J. Ullrich, M.D., Ph.D., ${ }^{4,5}$ R. Michael ScotT, M.D., ${ }^{3,4}$ \\ and Liliana C. Goumnerova, M.D. ${ }^{3,4}$ \\ ${ }^{1}$ Department of Neurosurgery, Washington University, St. Louis, Missouri; ${ }^{2}$ Department of Neurosurgery, \\ Ohio State University, Columbus, Ohio; Departments of ${ }^{3}$ Neurosurgery and ${ }^{5}$ Neurology, Boston Children's \\ Hospital; and ${ }^{4}$ Pediatric Neuro-Oncology Center, Dana-Farber Cancer Institute, Boston, Massachusetts
}

\begin{abstract}
Object. Low-grade glial and glioneuronal brain tumors are frequently encountered in the pediatric population and can be effectively treated by resection. The authors aimed to use imaging to evaluate how often tumors recurred and to determine if recurrences were associated with any clinical symptoms, along with the financial costs of imaging, in patients with radiographically proven gross-total resection (GTR) at Boston Children's Hospital. These data were assessed to propose guidelines regarding postoperative surveillance.

Methods. The authors performed a retrospective cohort analysis of the Pediatric Brain Tumor Program database from 1993 to 2003 to identify patients with glial or glioneuronal tumors initially evaluated at Boston Children's Hospital. Among the 888 patients evaluated for any type of brain tumor during this period, 67 patients had WHO Grade I glial or glioneuronal lesions with radiographically proven GTR and available follow-up data. The frequency and timing of postoperative imaging was compared with the institutional protocol. Recurrence-free survival was calculated using the Kaplan-Meier method. Financial costs of imaging were available from 2001 to 2009 and were averaged to extrapolate the postoperative surveillance costs.

Results. Among the 67 patients with GTR, 13 recurrences were detected radiographically with a mean time to recurrence of 32.4 months (range 2.9-128.5 months). The mean duration of follow-up after surgery was 6.6 years. The recurrence-free survival at 2 and 5 years after GTR for all low-grade glial and glioneuronal tumors was 0.90 (95\% CI 0.82-0.97) and 0.82 (95\% CI 0.73-0.92), respectively. No clinical symptoms were associated with any of the recurrences, and no deaths occurred. Under the institutional protocol of surveillance imaging, the estimated cost per recurrence at 5 years was $\$ 104,094$ per patient. The proposed protocol would reduce the number of MR scans in the first 5 years from 10 to 5, providing a potential cost savings of $\$ 52,047$ per recurrence.

Conclusions. Given the slow-growing, clinically asymptomatic nature of low-grade glial and glioneuronal tumors coupled with the financial and psychological costs of repeated imaging, the authors propose a postoperative surveillance MRI schedule that is less intensive than current institutional practice. (http://thejns.org/doi/abs/10.3171/2014.6.PEDS1321)
\end{abstract}

\section{KeY Words - glioma • pediatric brain tumor • pilocytic astrocytoma • magnetic resonance imaging $\bullet$ recurrence $\bullet$ oncology}

$\mathrm{L}$ OW-GRADE glial and glioneuronal tumors are among the most commonly encountered brain tumors in the pediatric population. Of these lesions, the WHO Grade I histological subtypes in particular carry an excellent prognosis and are generally amenable to surgical removal. Although these tumors are commonly viewed as

Abbreviations used in this paper: DNET = dysembryoplastic neuroepithelial tumor; GTR = gross-total resection; JPA = juvenile pilocytic astrocytoma. indolent, patients are frequently followed in the postoperative period with interval surveillance imaging to detect recurrence, with the concept that recurrences found earlier might be technically easier to resect and subsequently ameliorate or prevent clinical symptoms.

The literature describing patients who have undergone gross-total resection (GTR) of these low-grade tumors reveals a growing sense that frequent postoperative imaging may be of questionable utility due to the extremely low incidence of recurrence. Long-term rates of recur- 
rence following GTR for juvenile pilocytic astrocytomas (JPAs) of the cerebellum are reported to range from 0 to $14 \%$, and that for gangliogliomas (WHO Grade I) is only $1 \% .^{1,2,7,9,10,12-15}$ The GTR of dysembryoplastic neuroepithelial tumors (DNETs) also appears to be correlated with low recurrence rates and improved seizure control..$^{8,10,11}$ However, several of these studies were performed prior to the advent of MRI or with a substantial subset of patients followed by the less sensitive modality of CT. In the MRI era, a study of 40 pediatric patients following GTR of JPA reported a recurrence rate as high as $27.5 \% .^{4}$ Given the real incidence of recurrence following GTR, a rational schedule of follow-up surveillance MRI is necessary even long after the surgery. ${ }^{2,5}$ The utility of frequent interval postoperative imaging can also be evaluated in the context of the financial and psychological burden of MRI, which often requires conscious sedation or general anesthesia in young pediatric patients.

At Boston Children's Hospital, pediatric patients with WHO Grade I glial and glioneuronal tumors undergo frequent postoperative surveillance MRI. Using a brain tumor patient database covering all patients who were initially evaluated for a brain tumor at Boston Children's Hospital between 1993 and 2003, we asked the following questions: 1) Did patients with complete lesion resection exhibit recurrence on follow-up MRI? 2) Was radiographic recurrence associated with new neurological symptoms or a clinical intervention? The estimated financial cost of postoperative surveillance in terms of modern US dollars was also assessed. Based on these data, we propose a modified, less frequent schedule of postoperative MRI surveillance to follow these patients.

\section{Methods}

\section{Patients and Follow-Up}

The Pediatric Brain Tumor Program database (DanaFarber Cancer Institute) spanning 1993-2003 was retrospectively examined to identify patients who were diagnosed with a brain tumor during this period and received a histopathological diagnosis of WHO Grade I glial or glioneuronal tumors following biopsy or craniotomy. The clinical, radiographic, and histopathological data of identified patients were examined. Among the 888 total patients evaluated for any type of brain tumor during this period, 178 patients $(20 \%)$ had WHO Grade I glial or glioneuronal lesions as determined by histopathological examination. Of these 178 patients, craniotomy for complete tumor removal was attempted in 96 patients, with radiographically proven GTR in 84. Overall, GTR was achieved in $47.2 \%$ of all patients regardless of the preoperative surgical plan and in $87.5 \%$ of patients in whom complete removal was anticipated. Those patients who were found to have residual tumor on immediate postoperative MRI and who underwent a second craniotomy during the same admission were also considered to have undergone GTR if the second postoperative image demonstrated no residual tumor. A GTR was defined as lack of nodular enhancement on imaging performed in the immediate postoperative period (within 3 days of the surgery) by a neuroradiologist and 2 neurosurgeons. For nonenhancing tumors, such as DNETs, GTR was determined in a similar manner based on noncontrast imaging (FLAIR and T2-weighted) by a neuroradiologist and 2 neurosurgeons. Follow-up clinical and MRI data were available for 67 patients who underwent GTR. No deaths occurred in this population during the study period. Additionally, none of these patients received chemotherapy or radiation therapy. Cerebrospinal fluid diversion procedures were not examined as part of this study.

Preoperative and postoperative MR images were evaluated by staff neuroradiologists without prior knowledge of the extent of resection. During the study period, the routine postoperative MRI schedule at Boston Children's Hospital was as follows: immediately in the postoperative period, every 3 months during Year 1, every 6 months during Year 2, yearly until 5 years after surgery, and then thereafter every $2-3$ years. The financial costs of imaging and associated sedation/anesthetic procedures were available from October 1, 2001, until the end of follow-up on March 29, 2009. For imaging and sedation prior to 2001 and for follow-up performed at outside hospitals, the financial estimates of imaging and sedation were extrapolated by averaging the costs in US dollars from the available financial data. All children 5 years of age or younger were assumed to have undergone conscious sedation for the average durations of brain MR scans (approximately 35 minutes without and 60 minutes with sedation). Institutional review board approval was obtained for this study.

\section{Statistical Analysis}

The Kaplan-Meier method was used to calculate recurrence-free survival. Analyses were performed using Excel and SPSS software (Microsoft Inc. and IBM Inc., respectively).

\section{Results}

\section{Clinical Characteristics}

The clinical features of the 67 pediatric patients who underwent GTR of WHO Grade I glial and glioneuronal tumors and had available follow-up data are summarized in Table 1. The mean age at the time of craniotomy was 9.1 years (range 1-21.5 years), with a female predominance of approximately 1.4:1. In terms of tumor location, $63 \%$ were cerebellar and $24 \%$ were temporal. A histological diagnosis of JPA was made in the majority of patients $(69 \%)$, followed by ganglioglioma (21\%), DNET $(9 \%)$, and glioneuronal tumor $(1.5 \%)$. The mean duration of the follow-up period, including radiographic imaging, was 6.6 years, ranging between 1 and 14.7 years.

In this population, the total number of MR scans performed was 645 , with a total of 578 being obtained in the postoperative period. On average, an individual patient underwent 8.6 postoperative scans (median 9). In comparison, the average time to the last radiographic examination was 6.6 years (median 6.1 years), which should equate to approximately 9 postoperative surveillance scans according to the routine protocol at Boston Chil- 
A. H. Kim et al.

TABLE 1: Clinical and postoperative surveillance characteristics of 67 children with low-grade glial and glioneuronal tumors

\begin{tabular}{lc}
\hline \multicolumn{1}{c}{ Features } & Values \\
\hline age (yrs) & \\
mean & 9.1 \\
median & 8.0 \\
range & $1.0-21.4$ \\
sex ratio (M/F) & $28: 39$ \\
location, no. (\%) & \\
cerebellum & $41(61.2)$ \\
temporal & $16(23.9)$ \\
parietal & $4(6.0)$ \\
frontal & $2(3.0)$ \\
brainstem & $2(3.0)$ \\
occipital & $2(3.0)$ \\
tumor histology, no. (\%) & \\
JPA & $46(68.7)$ \\
ganglioglioma & $14(20.9)$ \\
DNET & $6(9.0)$ \\
glioneuronal & $1(1.5)$ \\
length of follow-up (yrs) & \\
mean & 6.6 \\
median & 6.1 \\
range & $1.0-14.7$ \\
total no. postop MR scans & 578 \\
no. of MR scans/patient & \\
$\quad$ mean & \\
mediange & 8.6 \\
estimated no. sedation procedures (based & 66 \\
scans prior to age 5) & \\
\hline & \\
\hline & \\
\hline & \\
\hline &
\end{tabular}

dren's Hospital. This difference suggests that, in practice, the institutional postoperative MRI protocol was generally followed but not strictly adhered to in this patient population. Variation in postoperative surveillance may be attributable to several factors, including physician preference, patient compliance, or follow-up at another institution.

\section{Radiographic Recurrence and Its Relationship to Clinical Symptoms and Management}

Table 2 summarizes the characteristics of patients who were found to have evidence of recurrence on postoperative surveillance imaging. In the total population, 13 recurrences were noted (19.4\%), with a mean time to recurrence of 32.4 months (range 2.9-128.5 months). None of the recurrences was associated with neurological symptoms. Additionally, all recurrences were small, with a maximal diameter of $1 \mathrm{~cm}$. Resection was performed in 10 of these patients, whereas recurrence was observed in 3 patients.

Recurrences were then evaluated in the context of
TABLE 2: Clinical characteristics of 13 children post-GTR with recurrent disease

\begin{tabular}{lc}
\hline \multicolumn{1}{c}{ Features } & Values \\
\hline recurrences, no. (\%) & $13(19.4)$ \\
symptomatic & 0 \\
2nd resection & $10(14.9)$ \\
observation & $3(4.5)$ \\
location, no. (\%) & \\
cerebellum & $7(53.8)$ \\
temporal & $3(23.1)$ \\
frontal & $2(15.4)$ \\
parietal & $1(7.7)$ \\
brainstem & $1(7.7)$ \\
tumor histology, no. (\% total, \% of subtype) & \\
JPA & $9(13.4,19.6)$ \\
ganglioglioma & $3(4.5,21.4)$ \\
DNET & $1(1.5,16.7)$ \\
glioneuronal & 0 \\
mos. to recurrence & \\
mean & 32.4 \\
median & 12 \\
range & $2.9-128.5$ \\
\hline
\end{tabular}

tumor location and histopathology. Most recurrences occurred in the cerebellum (7/13) followed by temporal, frontal, and parietal and brainstem locations, generally mirroring the relative distributions of tumors in the entire population. There were 9 recurrent JPAs, constituting $13.4 \%(9 / 67)$ of all low-grade tumors that were completely resected during the first admission and $19.6 \%(9 / 46)$ of all JPAs that were removed. The JPA recurrences occurred predominantly in the cerebellum (8/9), yielding an overall recurrence rate of $19 \%(8 / 42)$ for cerebellar JPAs following GTR. Analysis of recurrence for the other WHO Grade I tumors examined was limited due to small sample sizes. With this in mind, recurrent ganglioglioma and DNET represented $4.5 \%$ (3/67) and 1.5\% (1/67) of all tumors following GTR, respectively. Within each particular histopathological type, recurrence was observed in $21.4 \%$ (3/14) of gangliogliomas and $16.7 \%$ (1/6) of DNETs after GTR.

Kaplan-Meier curves were plotted for the cumulative recurrence-free survival following GTR for the overall population of 67 patients (Fig. 1 upper) and recurrencefree survival for patients with individual histopathological tumor types (Fig. 1 lower). Our data set contained only 6 DNETs and 1 glioneuronal tumor. Given the limited numbers, these 2 tumor types were not plotted as specific tumor types. The recurrence-free survival at 2 and 5 years after GTR for all low-grade glial and glioneuronal tumors was 0.90 (95\% CI 0.82-0.97) and 0.82 (95\% CI 0.73-0.92) respectively. For JPAs, the estimated 2- and 5-year recurrence-free survival was 0.87 (95\% CI $0.77-0.97)$ and 0.82 (95\% CI 0.7-0.93), respectively. Finally, using the limited data sets for ganglioglioma and DNET, the recurrence-free survival at 2 years was 0.93 
Recurrence after total resection of low-grade pediatric tumors
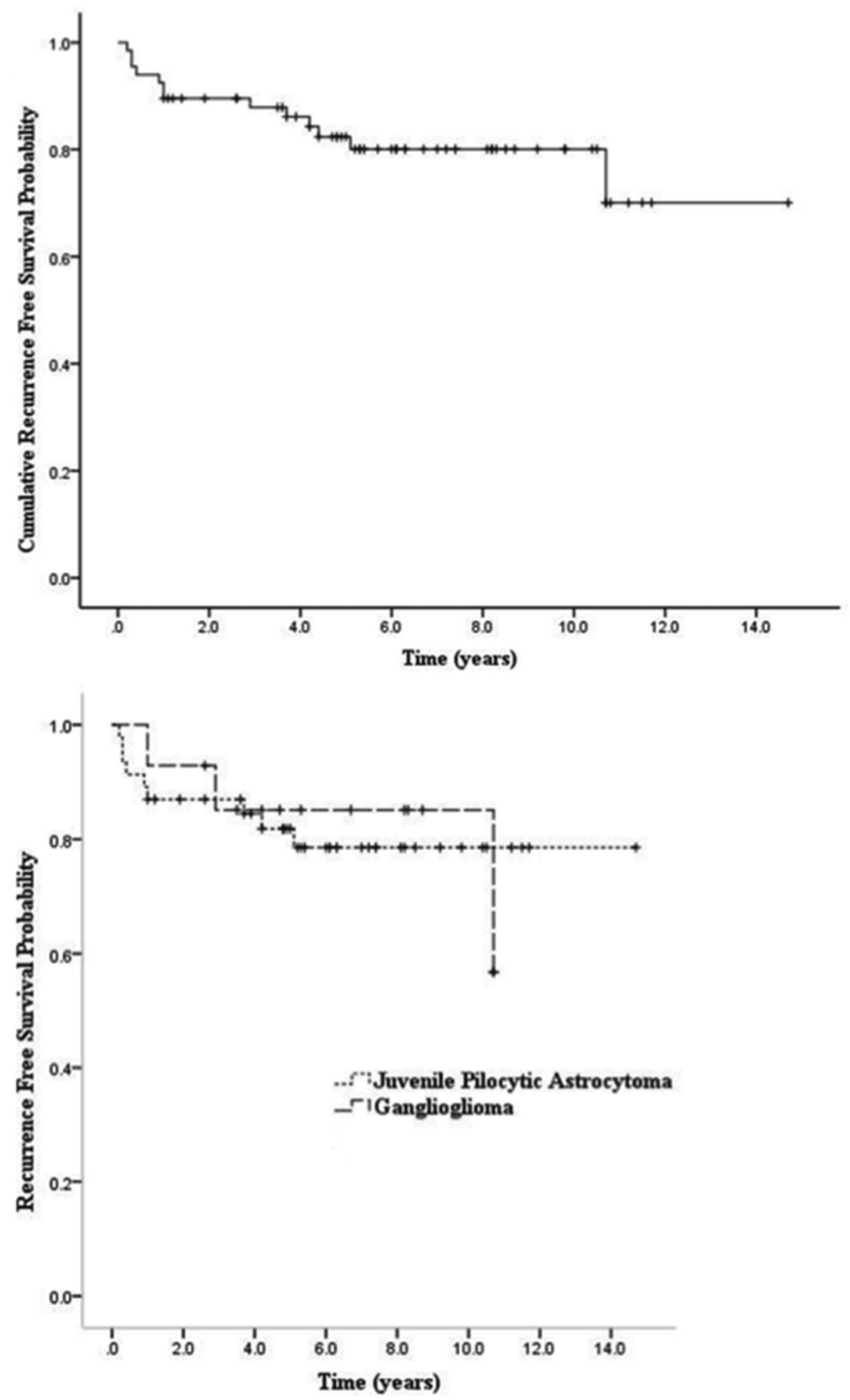

FIG. 1. Kaplan-Meier estimates of cumulative recurrence-free survival of all study patients (upper) and patients with JPAs and gangliogliomas (lower). 
(95\% CI 0.79-1.00) and 1.00 and at 5 years was $0.85(95 \%$ CI 0.66-1.00) and 0.67 (95\% CI 0.12-1.00), respectively.

We investigated the available images in our study patients to identify potential early radiographic markers of recurrence in patients with JPA or ganglioglioma, the largest 2 tumor subgroups in our population. Of the 67 patients in our study, 46 underwent GTR of a JPA or ganglioglioma and had immediate and early postoperative imaging (up to 12 months postoperatively), as well as later follow-up imaging, that we could examine (6 patients with recurrence and 40 with no recurrence). Among the patients in whom recurrence was observed, we found that nodular FLAIR signal in the tumor cavity on the immediate postoperative MR image that persisted to the first interval postoperative MR image might be a useful marker for recurrence. An example of this persistent nodular FLAIR signal that correlates with the future site of enhancing recurrent tumor is presented in Fig. 2. A preliminary univariate analysis of this particular radiographic finding among the subset of 46 patients (Table 3) revealed a significant correlation between persistent nodular FLAIR signal and recurrence defined by contrast enhancement at the same site (Fisher exact test, $\mathrm{p}<0.0216$ ). A major caveat of this analysis is that 2 of the 6 patients with recurrence showed evidence of recurrent tumor on the 3-month interval surveillance images; therefore, this persistent FLAIR signal on the 3-month MR scan was coincident with a contrast-enhancing nodule. Addition-
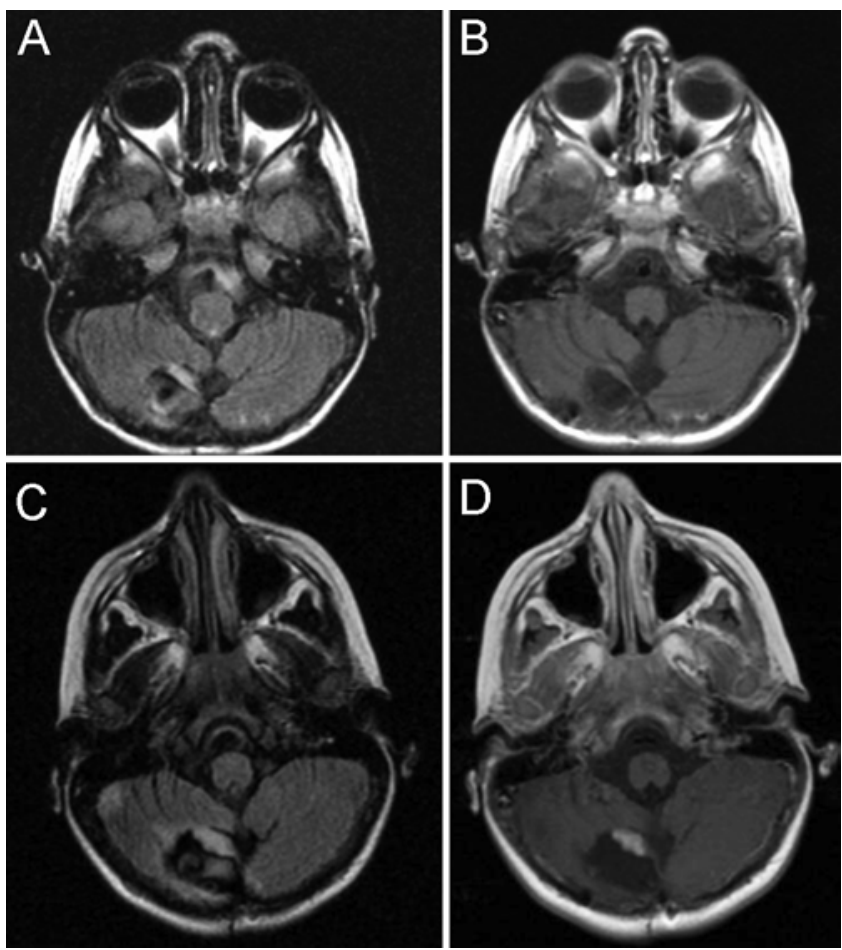

FIG. 2. Postoperative axial MR images of a pediatric patient following GTR of a JPA that recurred. The 5-month postoperative imaging study demonstrates persistent nodular FLAIR signal abnormality in the tumor cavity (A) in the absence of contrast enhancement (B). The 12-month postoperative MR image reveals a thickened FLAIR signal abnormality in the same location (C) with new overlapping contrast enhancement (D).
TABLE 3: Analysis of persistent nodular FLAIR signal and recurrence on postoperative MRI*

\begin{tabular}{ccc}
\hline Tumor & FLAIR + & FLAIR - \\
\hline recurrent & 6 & 0 \\
nonrecurrent & 17 & 23 \\
\hline
\end{tabular}

* + = positive; $-=$ negative.

ally, as seen in the group of patients without recurrence, persistent nodular FLAIR signal was a common finding $(42.5 \%)$ and thus was not highly specific. However, the absence of a persistent nodular FLAIR signal in the setting of GTR for JPA or ganglioglioma may be a useful prognostic marker for a lower likelihood of recurrence.

\section{Financial Estimates of Postoperative Surveillance Imaging}

To estimate the relative costs of postoperative MRI and associated anesthetic procedures, we averaged the actual financial data available for imaging and sedation performed on this patient population from 2001 to 2009 and rounded the costs to the nearest dollar. Actual financial data were available for 352 MR images, 180 radiology professional fees, and 345 contrast procedures charged to our patient population at Boston Children's Hospital during the study period. There were $7 \mathrm{MRI}$ studies acquired without contrast (average cost \$808, range \$583-\$998), 6 MR scans that included imaging of the spine (average cost \$1175, range \$1059-\$1212), and 3 MR scans obtained at an affiliated site (average cost \$1641, range \$1239-\$1818). The majority of MR scans (336) were acquired with and without contrast at an average cost of $\$ 1230$ at the same location (range \$1006-\$1542). The costs of MRI with and without contrast fluctuated over time throughout the study period in an inconsistent manner-in 2001 the mean cost $( \pm$ SD) was $\$ 1152$, and in 2009 the mean cost was $\$ 1064 \pm \$ 167$. Including all $352 \mathrm{MR}$ scans, the average cost was $\$ 1224 \pm \$ 175$. Considering an average radiology professional fee of $\$ 408$ and an average contrast cost of $\$ 77$ per MR scan, the average cost of MR with and without contrast, including professional fees and contrast, was $\$ 1709$. Extrapolating the average costs of these procedures to the study population of 67 patients, this would equate to roughly $\$ 1,102,305$ for the total number of MR scans with contrast (645 MR scans), including $\$ 987,802$ for postoperative imaging (578 MR scans). The financial data documented 59 sedation procedures, which added an additional $\$ 8732$. Sixteen patients were under the age of 5 years at the time of GTR, and there were 45 sedation and recovery charges. Financial data were not available for 2 patients who were under 5 years old at the time of GTR. In addition, financial data on professional fees for anesthesia, which represent a larger portion of the sedation cost for a visit, were not available. Thus, our actual sedation costs are likely an underestimate of the total costs of sedation and recovery in this patient population.

\section{Proposed Postoperative MRI Surveillance Schedule}

Given the lack of any clinical symptoms associated with recurrence after GTR in our patient population and 


\section{Recurrence after total resection of low-grade pediatric tumors}

the generally indolent growth characteristics of WHO Grade I glial and glioneuronal lesions, we propose a postoperative MRI surveillance schedule that is substantially less intensive than our current protocol. Although the absolute number of recurrences in our population was small, plotting the timing of recurrences provided a sense of when recurrences generally occur (Fig. 3). Recurrences appeared to cluster within year $1(n=7)$, between years 3 and $5(n=5)$, and at approximately 10 years $(n=1)$. Four of the recurrences occurred in the first 6 months at 2.9, $3.4,3.5$, and 4.8 months. We do not believe these early recurrences represented residual tumors, as the same criteria to define GTR were used to analyze postoperative imaging. In addition, a median time to recurrence of 6.4 months was previously reported following GTR for pilocytic astrocytomas. ${ }^{4}$ Therefore, we would recommend the following postoperative MRI schedule: (postoperative time = time 0) 1-3 days; and 3 months; and 1, 2, 5, and 10 years.

If this schedule were applied to our data set, assuming the actual time the recurrence was detected was the earliest radiographic sign of recurrence ( \pm 1 month), 6 of the recurrences would have been detected with no delay, and the remaining 7 recurrences would have been detected with delays ranging from 1.1 to 51.5 months, with an average delay of 9 months. However, it is highly likely that both the largest individual delays and the estimated average delay in detection are gross overestimates. The patient with the greatest hypothetical delay to detection using our proposed protocol showed the first radiological sign of recurrence at 128.5 months. However, no MR scan was obtained at 120 months, which may have reduced the detection delay. The next greatest hypothetical delay to detection of 25 months occurred for a patient who did not undergo the first follow-up surveillance MRI until postoperative Month 35. Similarly, a patient with a hypothetical 15.5-month delay to recurrence detection had surveillance MRI performed 2 weeks and 4 years postoperatively, with no intervening scans. Surveillance MRI at Month 24 in our proposed schedule may have identified both of these recurrences.
Clearly, individual patient clinical characteristics and needs should drive practice recommendations and define the rationale for this streamlined schedule of postoperative MR imaging. However, an additional benefit of this surveillance schedule is the substantial decrement in health care cost and the reductions in patient time and psychological burden. For a 10-year postoperative period, we compared the surveillance MRI costs of 3 different schedules: 1) the actual number of MR scans recorded for our data set, 2) the current practice of surveillance at Boston Children's Hospital (12 scans), and 3) our proposed schedule (6 scans). For our patient population, a total of 578 postoperative MR scans were obtained, with 566 scans acquired within a 10 -year postoperative period. This corresponds to an average of 8.4 scans per patient. Using our calculated average cost of an MR scan with contrast, based on actual financial data, 566 scans would cost approximately $\$ 967,294$. Based on current institutional practice, 12 contrast-enhanced MR scans per patient would cost approximately $\$ 1,374,036$. With the proposed surveillance schedule, the number of MR scans per patient would be reduced from 12 to 6 over a 10 -year period. For our patient population, the proposed schedule would provide a cost savings of $\$ 687,018$ over the current institutional schedule. In comparison with the actual number of MR scans and the current institutional practice, the proposed surveillance MRI schedule would achieve $26 \%$ and $50 \%$ reductions in costs, respectively. On a per patient basis for a 10-year surveillance period, the proposed schedule would cost $\$ 10,254$, which is equivalent to a savings of $\$ 4273$ per patient compared with estimated actual costs and a savings of $\$ 10,254$ per patient compared with the current institutional schedule.

Accounting for sedation and recovery costs based on patient age would provide additional savings. We assumed that all patients 5 years old or younger required conscious sedation for MRI. In our data set, 16 patients were under the age of 5 years at the time of GTR. Based on the number of surveillance MR scans acquired in these patients from the time of GTR to age 5, we inferred that 66 scans would have required sedation. Based on an

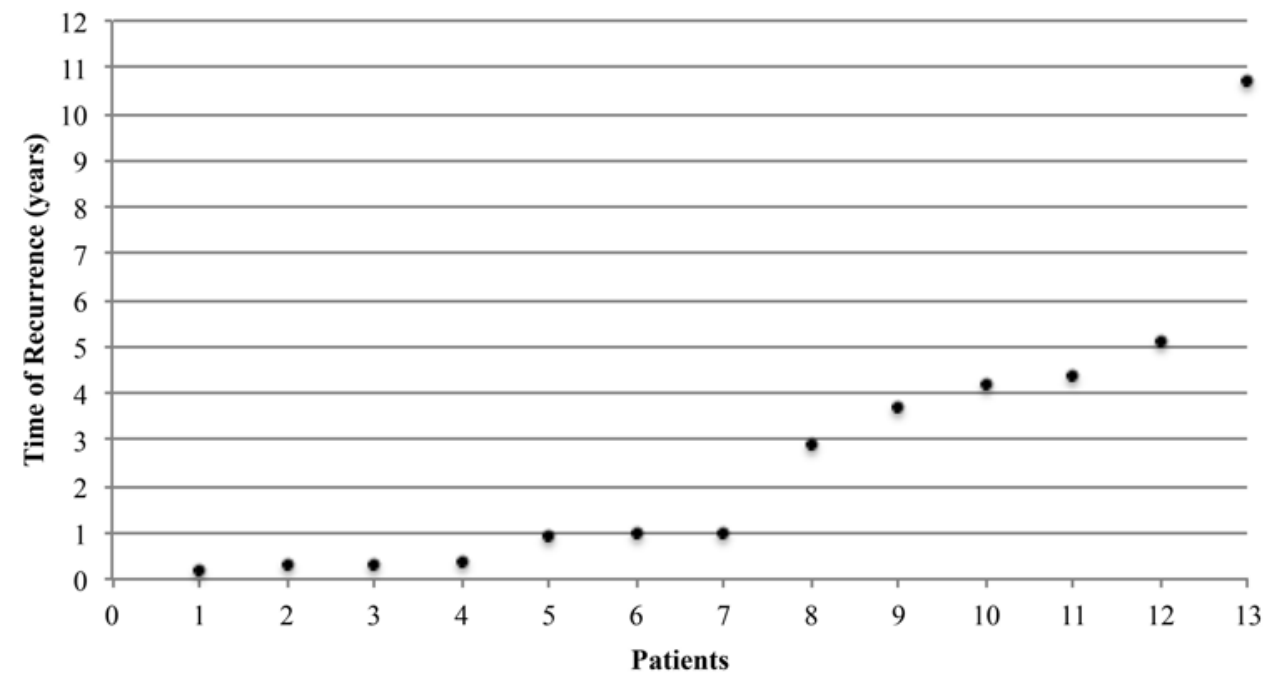

FIG. 3. Time point of recurrence for 13 children after GTR of low-grade glial tumors. 
average cost of $\$ 148$ per sedation, 66 sedation procedures would cost $\$ 9768$. Under the current schedule at Boston Children's Hospital, 97 postoperative scans would have been acquired with sedation in our patient population, whereas the number of postoperative MR scans with sedation would have decreased to 51 with the proposed plan. The decreased frequency of surveillance MR scans with sedation would reduce costs by $\$ 2960$ compared with the estimated number of sedation procedures based on the actual number of scans and $\$ 6808$ compared with current institutional practice.

Figure 4 plots the numbers of MR scans and recurrences over time. By Year 5, a total of $467 \mathrm{MR}$ scans were acquired, and 11 recurrences were detected. Based on the average cost of an MR scan with contrast, we were able to calculate a cost of $\$ 72,555$ per recurrence at 5 years. Under the current institutional schedule, at 5 years, 10 scans per patient would have been obtained for a total of 670 MR scans, which corresponds to a cost per recurrence of $\$ 104,094$. Under the proposed plan, only 5 scans per patient would have been acquired over the 5-year postoperative period, for a total of $335 \mathrm{MR}$ scans at an estimated cost per recurrence of $\$ 52,047$. The costs per recurrence under our proposed schedule represent reductions of $\$ 20,508$ and $\$ 52,047$, respectively, compared with the estimated cost per recurrence based on the actual and theoretical numbers of scans that would have been performed under the current institutional protocol.

\section{Discussion}

In this study, we investigated patients diagnosed with WHO Grade I glial and glioneuronal tumors between 1993 and 2003 to assess the post-GTR radiographic and clinically symptomatic recurrence rate of these tumors to recommend a rational protocol for postoperative surveillance MRI. As a group, these patients have an excellent prognosis following GTR, with estimated recurrence-free survivals of 0.90 (95\% CI 0.82-0.97) and 0.82 (95\% CI $0.73-0.92)$ at 2 and 5 years, respectively. A radiographic recurrence rate of $19.4 \%$ was observed, but all recurrences were clinically asymptomatic and small $(<1 \mathrm{~cm}$ in maximal diameter). In 3 of the 13 recurrences, the lesion was observed with interval imaging. Based on these data, we recommend a less frequent schedule of postoperative surveillance scanning as described above. The financial costs of brain MRI and associated sedation/anesthesia can be substantially decreased with this suggested protocol, although the savings will be heavily dependent on the age of the patient population and the tolerance of older pediatric patients to MRI procedures.

Other groups have suggested postoperative surveillance schedules for low-grade glial tumors following GTR, but they have published different opinions owing largely to divergent recurrence rates among studies. Several studies have documented a recurrence rate as low as $0 \%-2.5 \%$; Sutton and colleagues recommended no follow-up imaging for low-grade cerebellar astrocytomas after GTR. 1,6,7,14,15 However, the majority of these studies were carried out using patient data from the CT era, with a subset of patients followed by MRI in some cases, suggesting the possibility that these numbers might represent an underestimate of the actual recurrence rate. Others have reported higher post-GTR recurrence rates ranging from $6.5 \%$ to $27.5 \%$ among patients with Grade I lesions with recurrences identified 2-18 years after surgery. ${ }^{2,4,5,9}$ Indeed, a recurrence after GTR has been noted as late as 45 years after GTR based on the surgeon's opinion and

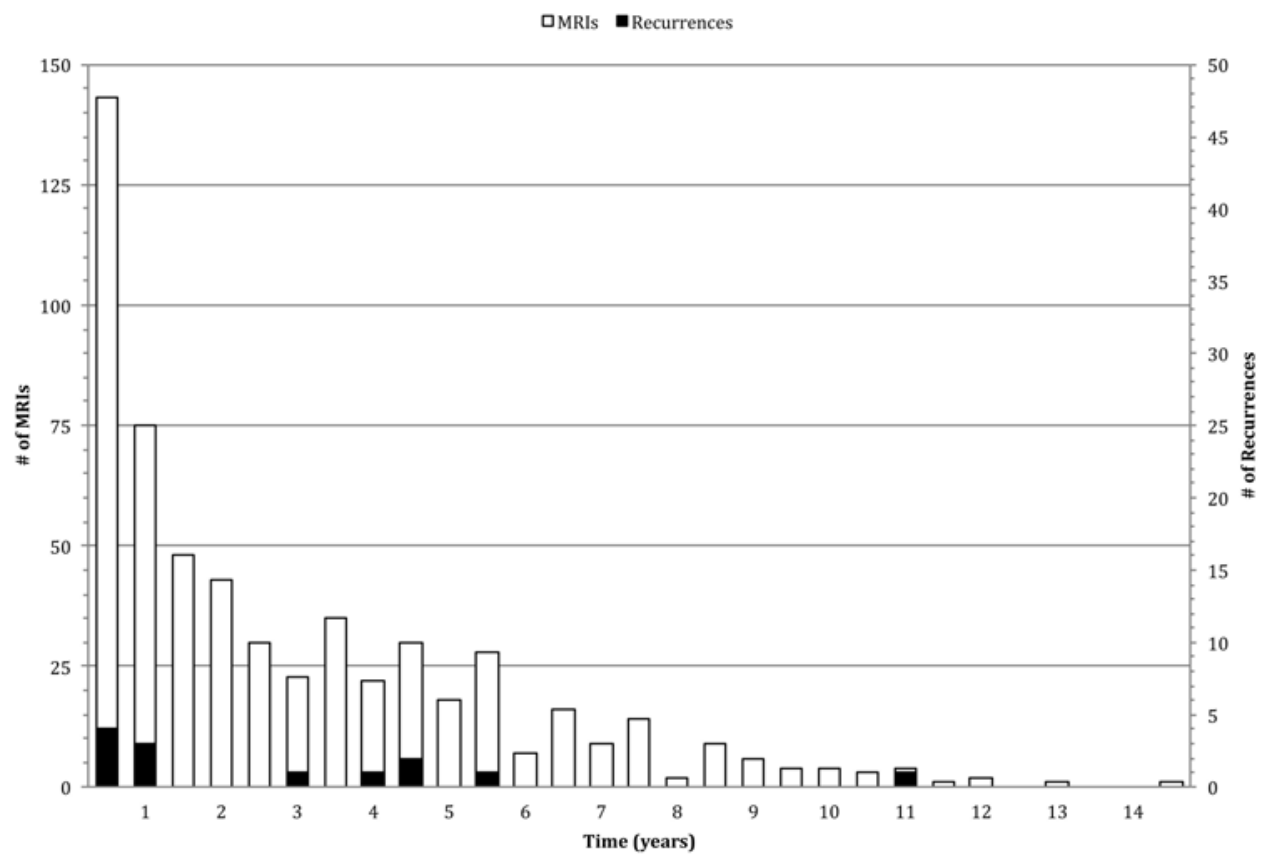

FIG. 4. Bar graph plotting the number of MR scans (unshaded) and recurrences (shaded) in 6-month periods. A cost per recurrence value can be estimated by comparing the number of MR scans and associated cost to the number of recurrences detected over time. 


\section{Recurrence after total resection of low-grade pediatric tumors}

MRI-detected recurrence. ${ }^{3}$ In particular, Benesch et al. voiced the need to follow up these patients radiographically despite GTR due to the possibility of recurrence after a long quiescent period. The patients in our study were all followed up by MRI and exhibited a low but significant rate of recurrence over a range of postoperative times (2.9-128.5 months), providing a persuasive rationale for continued surveillance imaging.

Significant remaining challenges include the development of guidelines for postoperative MRI surveillance beyond 10 years and the identification of factors that might predict future recurrence. Currently, we would recommend a 5-year surveillance schedule beyond 10 years. However, evidence-based recommendations are not possible at this time due to insufficient follow-up data beyond 10 years in the MRI era in pediatric patients with low-grade glial and glioneuronal tumors. An earlier report by Dorward and colleagues described early nodular enhancement at 3-6 months, high Ki 67 labeling index, and CD68 positivity as potential predictors of recurrence in patients with JPA following GTR. ${ }^{4}$ In our series, those patients with early nodular enhancement at 3-6 months were considered to have recurrent JPA, and 2 of these patients underwent repeat resection with pathologically proven evidence of tumor. In our preliminary analysis of radiographic features predictive of recurrence, we found that for JPAs and gangliogliomas, a persistent nodular FLAIR signal in the tumor cavity seen on both the immediate and first postoperative MR scan (within 12 months) may be predictive of recurrence. More strikingly, the absence of this radiographic finding may predict a very low likelihood of recurrence. These data also suggest that a patient's first interval postoperative MR scan, rather than the immediate postoperative scan, should be considered the patient's baseline postoperative study. Clearly, larger numbers of patients with adequate sequential MRI and multivariate analyses of recurrence are required to validate these preliminary findings. Future studies are needed to determine the appropriate frequency of long-term surveillance and to identify prognostic indicators that might predict recurrence among these patients.

\section{Conclusions}

World Health Organization Grade I glial and glioneuronal tumors can often be surgically managed, and cure rates can be excellent with lesions located in noneloquent brain tissue. In this study, craniotomy for complete tumor removal was attempted in 96 of 178 patients with histopathologically proven WHO Grade I glial or glioneuronal lesions, and radiographically proven GTR was achieved in 84 patients. Thus, GTR was achieved in $47.2 \%$ of all patients regardless of the preoperative surgical plan and in $87.5 \%$ of patients in whom complete removal was anticipated. Follow-up clinical and MRI data were available for 67 patients who underwent GTR, with an average follow-up time of 6.6 years.

The recurrence-free survival at 2 and 5 years after GTR for all low-grade glial and glioneuronal tumors was 0.90 (95\% CI 0.82-0.97) and 0.82 (95\% CI 0.73-0.92), respectively. No clinical symptoms were associated with any of the recurrences, and no deaths occurred.
However, given the small but real risk of recurrence even after GTR, we recommend a schedule of long-term postoperative surveillance MRI. Our recommended protocol has clear advantages over the current regimen at Boston Children's Hospital due to our finding that most recurrences in this group are clinically asymptomatic and generally indolent, allowing us to substantially decrease the number of MR scans necessary to safely follow up these patients. We recommend the following postoperative MRI schedule: (postoperative time = time 0) 1-3 days, 3 months, 1 year, 2 years, 5 years, and 10 years. This schedule would reduce the number of MR scans in the first 5 years following GTR from 10 to 5 . Based on the available financial data, the proposed schedule of postoperative MRI would provide a potential cost savings of $\$ 52,047$ per recurrence. The proposed schedule carries a clear financial benefit in terms of health care costs, and it may also lessen the psychological burden of frequent scans in this pediatric population.

\section{Acknowledgment}

We thank Marie Lightowler for her assistance in obtaining financial data for this study.

\section{Disclosure}

This project was funded in part by a Brain Science Foundation Fellowship Grant (A.H.K.).The authors report no conflict of interest concerning the materials or methods used in this study or the findings specified in this paper.

Author contributions to the study and manuscript preparation include the following. Conception and design: Goumnerova, Kim, Governale. Acquisition of data: Kim, Thompson, Santa. Analysis and interpretation of data: Goumnerova, Kim, Thompson, Santa, Cahill, Kieran. Drafting the article: Kim, Thompson, Chi, Ullrich, Scott. Critically revising the article: all authors. Reviewed submitted version of manuscript: all authors. Approved the final version of the manuscript on behalf of all authors: Goumnerova. Statistical analysis: Thompson, Cahill. Study supervision: Goumnerova.

\section{References}

1. Abdollahzadeh M, Hoffman HJ, Blazer SI, Becker LE, Humphreys RP, Drake JM, et al: Benign cerebellar astrocytoma in childhood: experience at the Hospital for Sick Children 19801992. Childs Nerv Syst 10:380-383, 1994

2. Benesch M, Eder HG, Sovinz P, Raith J, Lackner H, Moser A, et al: Residual or recurrent cerebellar low-grade glioma in children after tumor resection: is re-treatment needed? A single center experience from 1983 to 2003. Pediatr Neurosurg 42:159-164, 2006

3. Boch AL, Cacciola F, Mokhtari K, Kujas M, Philippon J: Benign recurrence of a cerebellar pilocytic astrocytoma 45 years after gross total resection. Acta Neurochir (Wien) 142:341346,2000

4. Dorward IG, Luo J, Perry A, Gutmann DH, Mansur DB, Rubin JB, et al: Postoperative imaging surveillance in pediatric pilocytic astrocytomas. Clinical article. J Neurosurg Pediatr 6:346-352, 2010

5. Due-Tønnessen BJ, Helseth E, Scheie D, Skullerud K, Aamodt G, Lundar T: Long-term outcome after resection of benign cerebellar astrocytomas in children and young adults (0-19 years): report of 110 consecutive cases. Pediatr Neurosurg 37:71-80, 2002

6. Fisher BJ, Leighton CC, Vujovic O, Macdonald DR, Stitt L: 


\section{A. H. Kim et al.}

Results of a policy of surveillance alone after surgical management of pediatric low grade gliomas. Int J Radiat Oncol Biol Phys 51:704-710, 2001

7. Garcia DM, Marks JE, Latifi HR, Kliefoth AB: Childhood cerebellar astrocytomas: is there a role for postoperative irradiation? Int J Radiat Oncol Biol Phys 18:815-818, 1990

8. Khajavi K, Comair YG, Wyllie E, Palmer J, Morris HH, Hahn JF: Surgical management of pediatric tumor-associated epilepsy. J Child Neurol 14:15-25, 1999

9. Krieger MD, Gonzalez-Gomez I, Levy ML, McComb JG: Recurrence patterns and anaplastic change in a long-term study of pilocytic astrocytomas. Pediatr Neurosurg 27:1-11, 1997

10. Luyken C, Blümcke I, Fimmers R, Urbach H, Elger CE, Wiestler OD, et al: The spectrum of long-term epilepsy-associated tumors: long-term seizure and tumor outcome and neurosurgical aspects. Epilepsia 44:822-830, 2003

11. Nolan MA, Sakuta R, Chuang N, Otsubo H, Rutka JT, Snead OC III, et al: Dysembryoplastic neuroepithelial tumors in childhood: long-term outcome and prognostic features. Neurology 62:2270-2276, 2004

12. Ogiwara H, Bowman RM, Tomita T: Long-term follow-up of pediatric benign cerebellar astrocytomas. Neurosurgery 70:40-48, 2012
13. Saunders DE, Phipps KP, Wade AM, Hayward RD: Surveillance imaging strategies following surgery and/or radiotherapy for childhood cerebellar low-grade astrocytoma. J Neurosurg 102 (2 Suppl):172-178, 2005

14. Sutton LN, Cnaan A, Klatt L, Zhao H, Zimmerman R, Needle $\mathrm{M}$, et al: Postoperative surveillance imaging in children with cerebellar astrocytomas. J Neurosurg 84:721-725, 1996

15. Vassilyadi M, Shamji MF, Tataryn Z, Keene D, Ventureyra E: Postoperative surveillance magnetic resonance imaging for cerebellar astrocytoma. Can J Neurol Sci 36:707-712, 2009

Manuscript submitted January 16, 2013.

Accepted June 17, 2014.

Portions of this work were presented in abstract form as proceedings at the Annual Meeting of the American Association of Neurological Surgeons, Philadelphia, PA, May 3, 2010.

Please include this information when citing this paper: published online July 25, 2014; DOI: 10.3171/2014.6.PEDS1321.

Address correspondence to: Liliana C. Goumnerova, M.D., Boston Children's Hospital, 300 Longwood Ave., Hunnewell 2, Boston, MA 02115.email: liliana.goumnerova@childrens.harvard.edu. 